SECTION 31. Economic research, finance, innovation, risk management.

Anatoly Aleksandrovich Naumov

Docent, Candidate of Technical Sciences,

Center of Applied Mathematical Research, Novosibirsk, Russia,

E-mail: $\underline{\text { a_a_naumov@mail.ru }}$

\title{
FUZZY PROJECTS: MODELS AND ALGORITHMS
}

Abstract: The paper discusses the modern models and algorithms for solving fuzzy projects analysis problems.

Keywords: Investment projects, fuzzy sets, fuzzy projects, risks, models, algorithms.

\section{УДК 330.322.5}

\section{РАЗМЫТЫЕ ПРОЕКТЫ: МОДЕЛИ И АЛГОРИТМЫ}

Аннотация: В работе рассмотрены современные модели и алгоритмы решения задач анализа размытых проектов.

Ключевые слова: Инвестиционные проекты, размытые множества, размытые проекты, риски, модели, алгоритмы.

Работа представляет собой обзор современных методов анализа размытых проектов и является продолжением работы [1].

Неопределенности инвестиционных проектов (ИП) могут быть описаны многими способами. Известно, что при этом преобладают вероятностные модели, они же чаще всего используются и при оценивании рисков. Вероятностные модели являются фундаментальной основой для решения задач анализа ИП на эффективность. Однако вероятностные модели не всегда могут хорошо описать некоторые виды неопределенностей, возникающих на практике. Другие модели описания неопределенностей, такие, например, как нечеткие множества [2; 3], позволяют явно рассмотреть основные причинно-следственные связи и адекватно представить неопределенность сложных объектов, к каким относятся и ИП. Если функции распределения параметров ИП заданы неточно (т.е. присутствует неопределенность в их задании), то обычно применяют аналогичные функции, построенные на теории размытых множеств. В данной работе предполагается, что и размытая функция плотности вероятностей параметров проекта задана неточно.

В работах [4; 6-8] рассмотрены вопросы использования размытых множеств при анализе кредитных лизинговых проектов и проектов банков, когда неопределенности относятся к временам активностей проектов. При этом анализируется изменение длительности критического пути проекта.

В работах [13; 14] использованы размытые множества при анализе проектов, когда неопределенности относятся как к временам активностей, так и к структуре проектов.

Задачи планирования проектов при размытых ограничениях на ресурсы (FRCPSP) и выравнивания размытых ресурсов (FRLP) рассмотрены в работе [15]. В ней для решения FRCPSP и FRLP задач использованы Greedy-алгоритм и генетический алгоритм. Приведен пример использования алгоритмов при анализе проектов по обслуживанию гражданских вертолетов в рамках французского промышленного проекта под названием Helimaintenance. 
В работе [16; с. 277-320] рассмотрены вопросы применения пакета программ MatLab для решения задач использования размытых множеств с использованием экспертов при проектировании сетей.

Риски инвестиционных проектов (ИП), представимых в виде потоковых моделей $\overparen{B P}_{s}(t)[17]$, обусловлены неопределенностями в знании значений их параметров $\pi$. Очевидно, такая неопределенность в знании характеристик ИП приводит к тому, что вместо вектора плановых показателей эффективности $\vec{Q}$ реально будем иметь дело с вектором $\overrightarrow{\widetilde{Q}}$. Величина отклонения вектора $\vec{Q}$ от вектора $\overrightarrow{\widetilde{Q}}$ и будет характеризовать риск ИП. Процедура оценивания рисков может быть представлена отображением:

$$
\langle\widetilde{B P}, \tilde{\pi}\rangle \stackrel{\varphi_{R}}{\longrightarrow} \vec{R}(t),
$$

где $\varphi_{R}$ - оператор оценивания рисков. Вектор $\vec{R}(t)$ включает в себя как внутренние, так и внешние риски.

При моделировании и оценивании значений показателей $\overrightarrow{\widetilde{Q}}(t)$ используются характеристики неопределенности ИП: $f(\tilde{\pi})$ - плотность вероятностей возмущенных значений множества параметров $\pi, \mu_{\grave{A}}(\tilde{\pi}) \cdot f(\tilde{\pi})$ - плотность вероятностей для размытого множества параметров. Здесь $\mu_{\dot{\hat{A}}}(\tilde{\pi})$ - функция принадлежности, $A \subseteq \Pi, \Pi-$ область допустимых значений параметров $\tilde{\pi}[1]$.

Предлагается неопределенность в знании точного вида этих функций представить в виде множеств их допустимых значений: $f(\tilde{\pi}) \in \mathrm{F}_{f}(\tilde{\pi})$ и $\mu_{\dot{\AA}}(\tilde{\pi}) \in \mathrm{F}_{\mu}(\tilde{\pi})$. Например, множества $\mathrm{F}_{f}(\tilde{\pi}) \quad$ и $\quad \mathrm{F}_{\mu}(\tilde{\pi}) \quad$ могут быть дискретными $\left(\mathrm{F}_{f, N_{f}}(\tilde{\pi})=\left\{f_{1}(\tilde{\pi}), f_{2}(\tilde{\pi}), \ldots, f_{N_{f}}(\tilde{\pi})\right\}\right)$ или параметризованными так, что при изменении параметров элементы этих множеств принимают все допустимые значения из области неопределенности $\left(\mathrm{F}_{\mu, \theta}(\tilde{\pi})=\left\{\mu_{\dot{A}}(\tilde{\pi}, \theta) \mid \theta \in \Theta\right\}\right)$. Тогда, в результате моделирования в соответствии с множествами функций неопределенности $\mathrm{F}_{f, N_{f}}(\tilde{\pi})$ или $\mathrm{F}_{\mu, \theta}(\tilde{\pi})$ будут получены пары «показатели эффективности - риски» вида $Q_{i R}=\left\langle Q_{i, \hat{i} \hat{i} i} ;\left\{R_{j, i}^{(l)}\right\}, j=1,2, \ldots, K, l=1,2, \ldots, N_{f}\right\rangle, \quad i \in\{1,2, \ldots, M\} \quad$ для $\quad \mathrm{F}_{f, N_{f}}(\tilde{\pi}) \quad$ и $Q_{i R}=\left\langle Q_{i, \hat{i} i \grave{i}} ;\left\{R_{j, i}(\theta)\right\}, j=1,2, \ldots, K, \theta \in \Theta\right\rangle, \quad i \in\{1,2, \ldots, M\}$ для $\mathrm{F}_{\mu, \theta}(\tilde{\pi})$. Остается только свернуть множество рисков, оцененных для показателей эффективности, в одно значение.

Например, если риски оцениваются наибольшими возможными потерями, то свертки могут иметь такой вид: $R_{j_{0}, i}^{(\max )}=\max _{l=1,2, \ldots, N_{f}} R_{j_{0}, i}^{(l)}$ для $\mathrm{F}_{f, N_{f}}(\tilde{\pi})$ и $R_{j_{0}, i}^{(\max )}=\max _{\theta \in \Theta} R_{j_{0}, i}(\theta)$ для $\mathrm{F}_{\mu, \theta}(\tilde{\pi})$. И окончательно получим оценки: $Q_{i R}=\left\langle Q_{i, \hat{i} i \hat{i}} ;\left\{R_{j_{0}, i}^{(\max )}\right\}\right\rangle, \quad i \in\{1,2, \ldots, M\}$. Здесь индекс $j_{0} \in\{1,2, \ldots, K\}$ и соответствует риску наибольших возможных потерь.

Более подробно с видами рисков и алгоритмами их оценивания можно ознакомиться в работе [17]. 


\section{References:}

1. Наумов А.А. Использование размытых множеств при оценивании рисков инвестиционных проектов/ А.А. Наумов, С.У. Жанатауов// ISJ Theoretical\&Applied Science, Materials of the ISPC «Computer technologies in science», 30.04.2014, Valencia, Venezuela, 2014, № 4 (12), C. 178-181. URL: https://sites.google.com/site/anatolynaumov2011/home/spisok-trudov-list-of-papers .

2. Zadeh L.A. From imprecise to granular probabilities// Fuzzy Set Systems, 2005, Vol. 154, pp. 370-374.

3. Zimmermann H-J. An application-oriented view of modelling uncertainty// European Journal of Operation Research, 2000, Vol. 122, pp. 190-199.

4. Chen Y.C. A study on the quality of credit granting in leasing: fuzzy set theory approach// Soft Computing, 2001, 5, pp. 45-59.

5. Dowd K. A moments-based procedure for evaluating risk forecasting models// In: The Analytics of Risk Model Validation, Ed. Christodoulakis G., Satchell S. - Oxford: Elsevier, 2008, pp. 45-58.

6. Fiordelisi F., Marques-Ibanez D., Molyneux P. Efficiency and risk in European banking// In: Working Paper Series, European Central Bank, 2010, June. 37.

7. Vasiliauskaite A., Cvilikas A. Fuzzy sets theory adoption for credit risk assessment in leasing sector// Taikomoji ekonomika: sisteminiai tyrimai = Applied economics: systemic research, 2008, 2, pp. 85-98.

8. Paužuolis V., Cvilikas A. Fuzzy sets theory for leasing's credit risk assessment: empirical evidence// European Journal of Business and Social Sciences, 2014, Vol. 2, No. 12 , pp. 1-14. URL: http://www.ejbss.com/recent.aspx.

9. Chanas S., Zielinski P. Critical Path Analysis in the Network with Fuzzy Activity times// Fuzzy Set and Systems, 2001, Vol. 122, pp. 195-204.

10. Chen S.P. Analysis of Critical Paths in a Project Network with Fuzzy Activity times// European Journal of Operational Research, 2007, Vol. 183, pp. 442-459.

11. Zammori A.Z., Braglia M., Frosolini M. A Fuzzy Multi-Criteria Approach for Critical Path Definition// International Journal of Project Management, 2009, Vol. 27, pp. 278291.

12. Bevilacqua M., Ciarapica F.E., Giacchetta G. Critical Chain and Risk Analysis Applied to High-Risk Industry Maintenance: A Case Study// International Journal of Project Management, 2009, Vol. 27(4), pp. 419-432.

13. Morovatdar R., Aghaie A., Haji Yakhchali S. Fuzzy Network Analysis for Projects with High Level of Risks - Uncertainty in Time and Structure// International Journal of Industrial Engineering \& Production Research, 2011, Vol. 22, No. 1, pp. 73-82.

14. Arunraj N.S., Mandal S., Maiti J. Modeling uncertainty in risk assessment: An integrated approach with fuzzy set theory and Monte Carlo simulation// Accident Analysis \& Prevention, 2013, Vol. 55, pp. 242-255.

15. Masmoudi M., Hait A. Project scheduling under uncertainty using fuzzy modelling and solving techniques// Engineering Applications of Artificial Intelligence, 2013, Vol. 26, N 1, pp. 135-149.

16. Sivanandam S.N., Sumathi S., Deepa S.N. Introduction to Fuzzy Logic using MATLAB. - Berlin: Springer, 2007.

17. Наумов А.А. Методы анализа и синтеза инвестиционных проектов. Эффективность, риски, управление. - LAP LAMBERT Academic Publishing, 2013. 356 c. URL: https://www.ljubljuknigi.ru/store/ru/book/Методы-анализа-и-синтезаинвестиционных-проектов/isbn/978-3-659-51240-7 . 